\title{
Práticas assistenciais em reanimação do recém-nascido no contexto de um centro de parto normal*
}

\author{
CARE PRACTICES ON THE RESUSCITATION OF NEWBORNS \\ INTHE CONTEXT OF ADELIVERY CENTER
}

\author{
PRÁCTICAS ASISTENCIALES EN REANIMACIÓN NEONATAL \\ EN EL CONTEXTO DE UN CENTRO DE PARTO NORMAL
}

\author{
Karina Fernandes ${ }^{1}$, Amélia Fumiko Kimura²
}

\begin{abstract}
RESUMO
Aproximadamente 10 a $15 \%$ dos recém-nascidos (RNs) apresentam dificuldades de adaptação ao nascimento, o que requer habilidade e prontidão dos profissionais para intervir nessas situações. Este estudo observacional, transversal objetivou descrever as práticas assistenciais empregadas em reanimação neonatal em um Centro de Parto Normal de um hospital público de São Paulo. Observou-se 100 atendimentos prestados pela equipe profissional e os dados foram registrados em um instrumento check-list. A presença de líquido meconial foi constatada em 24 (24,0\%) partos e a aspiração das vias respiratórias foi realizada em $47(47,0 \%)$ RNs. Desse total, 3 $(6,4 \%)$ tiveram a traquéia aspirada e $26(26,0 \%) \mathrm{RNs}$ receberam oxigenação, sendo que $5(19,2 \%)$ com máscara aberta e pressão positiva. Massagem cardíaca foi realizada em $1(1,0 \%)$ RN. Após a reanimação neonatal inicial, 6 $(6,0 \%) \mathrm{RNs}$ foram transferidos à UTI neonatal devido desconforto respiratório.
\end{abstract}

\section{DESCRITORES}

Ressuscitação cardiopulmonar. Recém-nascido. Enfermagem neonatal. Parto.

\begin{abstract}
Approximately 10 to $15 \%$ of the neonates present difficulties in adapting to extra uterine life, which requires ability and readiness from the professional to intervene properly. This is an observational, cross-sectional study aimed at describing the care practices on the resuscitation of the newborn in the Delivery Center of a public hospital in the city of São Paulo. Data were obtained from the observation of 100 assistances given by health care professional staff and were recorded on a check-list instrument. Meconium-amniotic fluid was found in 24 (24.0\%) births and $47(47.0 \%)$ newborns were submitted to respiratory airways aspiration. Of these, 6.4\% (3) had their trachea aspirated. 26 (26.0\%) newborns were ventilated, $5(19.2 \%)$ of which by mask plus positive pressure; 1 (1.0\%) newborn was submitted to a chest compression. After the initial resuscitation procedures, 6 (6.0\%) newborns were referred to the neonatal intensive care unit.
\end{abstract}

\section{KEY WORDS}

Cardiopulmonary resuscitation. Infant, newborn.

Neonatal nursing. Parturition.

\section{RESUMEN}

Aproximadamente entre 10 a $15 \%$ de los recién nacidos ( $\mathrm{RNs}$ ) presentan dificultades de adaptación al nacer. Así, el momento del nacimiento requiere habilidad y prontitud de los profesionales para intervenir de modo adecuado. Este estudio observacional, transversal realizado en un hospital público de São Paulo, tuvo como objetivo describir las prácticas asistenciales en reanimación neonatal en un centro de parto normal. Se observaron 100 atenciones al nacimiento prestados por el equipo profesional cuyos datos fueron registrados en un instrumento check-list. Se verificó presencia de líquido meconial en $24(24.0 \%)$ de las atenciones. Las vías respiratorias de $47(47.0 \%)$ RNs fueron aspiradas. De ese total, 3(6.4\%) tuvieron aspiración de tráquea. $26(26.0 \%) \mathrm{RNs}$ fueron oxigenados, 5(19.2\%) recibieron, además de la máscara abierta, ventilación por presión positiva. El masaje cardiaco fue empleado en 1(1.0\%) RN. Después de la reanimación neonatal 6(6.0\%) RNs fueron transferidos a la26(26.0\%) RNs UCI neonatal.

\section{DESCRIPTORES}

Resuscitación cardiopulmonar.

Recién nacido.

Enfermeíra neonatal.

Parto.
* Extraído da dissertação "Práticas assistenciais no atendimento imediato ao recém-nascido em Centro de Parto Normal", São Paulo, 2004

1 Enfermeira obstetra. Mestre em Enfermagem Obstétrica e Neonatal. Diretora Técnica de Saúde do Hospital Geral de São Mateus - São Paulo - SP fernandeskarina@uol.com.br

2 Enfermeira Obstetra. Professora Doutora do Departamento de Enfermagem Materno-Infantil e Psiquiátrica da Escola de Enfermagem da USP. fumiko@usp.br 


\section{INTRODUÇÃO}

Imediatamente após o nascimento, o neonato necessita assumir suas funções vitais que, durante a vida intra-uterina, eram realizadas pela placenta. $\mathrm{O}$ nascimento é considerado uma fase crítica, denominada de período de transição, que exige adaptações fisiológicas repentinas e cruciais no sistema corporal. Em essencial, os sistemas cardiovascular e pulmonar sofrem alterações assim que o cordão é clampeado, e tem início a respiração. $O$ período de transição fetal para o neonatal representa uma das fases mais dinâmicas e difíceis do ciclo vital humano que demanda a transformação de uma condição de completa dependência para outra de auto-suficiência em relação à oxigenação e nutrição ${ }^{(1-2)}$.

Em cerca de 85 a $90 \%$ dos nascimentos, a adaptação do RN do ambiente intra para o extra-uterino ocorre em um período rápido, de maneira fisiológica, atingindo a estabilização. Porém, alguns recémnascidos podem apresentar intercorrências e os profissionais necessitam estar habilitados para reconhecer precocemente e intervir nestas situações ${ }^{(2)}$.

Assim, os profissionais passam a ser coadjuvantes do processo do nascimento e as práticas "rotineiras" ou protocolares a todos os atendimentos devem ser evitadas, pois se não forem respeitadas, incorrerão em intervenções desnecessárias e prejudiciais ou mesmo iatrogênicas tanto à mulher como a seu concepto/recém-nascido (RN).

É fundamental que haja capacitação técnica dos profissionais que participam do atendimento imediato ao $\mathrm{RN}$, além de maior sensibilidade por parte dos profissionais voltados para uma assistência humanista, pois, em função do modelo assistencial no qual os profissionais foram inseridos durante sua formação, muitas "rotinas" assistenciais, que eram realizadas, pautavam-se no aspecto prático para facilitar o "bom andamento do serviço" e influenciavam o conhecimento e a prática assistencial desses profissionais ${ }^{(3)}$.

As condições de nascimento do RN podem estar relacionadas à qualidade do atendimento à mulher durante a gravidez, trabalho de parto e parto.

No Brasil, entre 1990 e 1995, os óbitos fetais tardios contribuíram com mais de 50,0\% na taxa de mortalidade perinatal, em 1995, a mortalidade perinatal contribui com $63 \%$ dos óbitos em crianças menores de um ano. Tais dados são de difícil interpretação por se desconhecer a magnitude da subnotificação de óbitos fetais e neonatais precoces, entretanto, é impossível ignorar a gravidade do problema da mortalidade perinatal. A maioria das mortes incide no período neonatal precoce, na primeira semana de vida, especialmente, no primeiro dia de vida, representando quase $36,0 \%$ das mortes. A taxa de mortalidade neonatal é resultado de complexas relações dos fatores biológicos, sociais e de assistência à saúde, o que torna sua redução mais difícil ${ }^{(4)}$. Dados de 1988 e 1992 demonstram que, dois terços dos óbitos infantis acontecem no período neonatal e cerca de $50,0 \%$ dos óbitos no primeiro ano de vida ocorrem na primeira semana de vida, ou seja, no período neonatal precoce ${ }^{(5-6)}$. Vale ressaltar que estudos epidemiológicos sobre morbidade e mortalidade neonatal envolvendo as causas e os fatores associados são escassos no Brasil, o que pode ser justificado, conforme já destacado, pela complexidade dos fatores envolvidos, o que dificulta o seu estudo.

O aumento da mortalidade neonatal também se verifica nos países desenvolvidos, porém existem diferenças importantes entre os países ricos e os em desenvolvimento( ${ }^{(7)}$. Nos países desenvolvidos, a principal causa de óbito neonatal é a má formação congênita, ao passo que no Brasil verifica-se que as principais causas são as perinatais. Após o nascimento, a primeira semana de vida é um dos períodos mais crítico. Estudo realizado em Porto Alegre demonstrou que $63,4 \%$ dos óbitos neonatais ocorreram nessa fase. Conclui-se que estes óbitos estão relacionados com a qualidade da assistência no pré-natal, parto e atendimento neonatal, sobretudo na sala de parto.

Mais da metade dos óbitos neonatais ocorrido no período de 1979 a 1996, no município de São Luís, capital do Estado do Maranhão, foi classificada pela Fundação SEADE como causas reduzíveis. Entre as causas citadas está a asfixia perinatal, considerada evitável, desde que se implemente um adequado atendimento ao parto e ao $\mathrm{RN}^{(8)}$.

A causa de morte neonatal por asfixia é um problema assistencial que implica questionar a qualidade do atendimento perinatal, no que se refere à disponibilidade dos recursos tecnológicos, da competência técnica e ética da equipe ${ }^{(8-9)}$.

Considerando a necessidade de garantir acesso ao parto nos serviços de saúde do Sistema Único de Saúde (SUS), o Ministério da Saúde (MS), em agosto de 1999, instituiu a Portaria ${ }^{\circ}$ 985/GM criando o Centro de Parto Normal (CPN) para atendimento ao parto normal sem distócia, prestando assistência humanizada e de qualidade à gestante, puérpera e RN. Na sua elaboração, a Portaria considerou prioridade a redução da mortalidade materna e perinatal por causas evitáveis, a necessidade de humanizar a assistência obstétrica e neonatal no 
âmbito do SUS e melhorar a qualidade da assistência na gestação parto e pós-parto ${ }^{(10)}$. Com base nessa Portaria, diversos serviços foram implantados no Brasil a partir de 1999.

Posteriormente, o MS em conjunto com a Federação Brasileira das Sociedades de Ginecologia e Obstetrícia - FEBRASGO e Associação Brasileira de Obstetrizes e Enfermeiros Obstetras - ABENFO lançou um manual intitulado Parto, aborto e puerpério: assistência humanizada à mulher ${ }^{(11)}$. Assim, neste manual, a "assistência humanizada" é definida como um conceito amplo que envolve conhecimento, práticas e atitudes, que têm como fim promover o parto e o nascimento saudáveis, prevenindo a morbimortalidade materna e perinatal. Inicia-se no pré-natal e procura garantir que os procedimentos realizados sejam benéficos ao binômio mãe-filho, evitando intervenções desnecessárias e preservando a privacidade e autonomia dos usuários.

Este manual, considerado pelos profissionais como um guia prático para atuação na assistência obstétrica, tem sido objeto de discussão no sentido de se rever as práticas, tradicionalmente adotadas e, ao mesmo tempo, servindo como metas a serem alcançadas pelos serviços.

A transformação do modelo assistencial hegemônico vigente não se consolida rapidamente, pois isso requer mudança de paradigma, crenças, atitudes e práticas que não depende apenas da qualificação técnica dos profissionais diretamente envolvidos em sua implementação, mas, também, das estruturas micro e macro-assistenciais envolvidas.

Como enfermeira obstétrica atuando em CPN de um hospital público estadual de nível secundário, situado na cidade de São Paulo, cuja proposta é oferecer um atendimento humanizado ao parto e nascimento, temos questionado se a introdução desta proposta inovadora tem resultado na redução dos índices de morbimortalidade materna e perinatal nos atendimentos realizados em CPN quando comparados aos resultados obtidos nos serviços tradicionais, conhecidos como Centro Obstétrico. Também é preciso verificar o impacto da introdução dessas práticas na modificação do padrão de atendimento, no que diz respeito às práticas assistenciais obstétricas e neonatais no contexto do CPN.

A assistência ao parto e nascimento em condições seguras exige infra-estrutura material e recursos humanos, que desenvolva trabalho em equipe interdisciplinar com profissionais treinados e integrados para atender a situações rotineiras e emergenciais.
Busca bibliográfica realizada nas bases de dados eletrônicas que catalogam artigos científicos nacionais e internacionais com abordagem na assistência imediata ao RN, constatou que este tema não tem sido objeto amplamente estudado. Assim, o que se observa é que na prática clínica o modelo assistencial de Centro de Parto Normal vem sendo implementado em nosso meio, entretanto, a literatura produzida sobre caracterização do atendimento ao recém-nascido atendido em CPN e sobre reanimação neonatal é escassa. Assim, obter dados que caracterizam as práticas de reanimação neonatal que vem sendo empregadas no atendimento ao $\mathrm{RN}$ em CPN para compará-los às práticas adotadas no modelo tradicional de centro obstétrico, trará subsídios para avaliar os resultados perinatais nesses modelos assistenciais.

Os RNs normais constituem a maioria dos nascimentos. O Ministério da Saúde recomenda que em toda sala de parto deve estar presente um profissional capacitado para realizar reanimação rápida e efetiva, mesmo nas situações em que se espera o nascimento de um bebê saudável. Com os RNs normais nada mais deve ser realizado além de enxugá-los, aquecê-los, avaliar as suas condições e entregá-los às suas mães para estabelecer um contato precoce $\mathrm{e}$ íntimo. As manobras de reanimação devem ser realizadas apenas em situações necessárias, sendo desaconselhável empregar estes procedimentos de forma rotineira ${ }^{(11)}$.

Os procedimentos realizados durante a reanimação neonatal consistem em manter a permeabilidade das vias aéreas, posicionando a cabeça e o pescoço do RN, aspiração da boca e nariz e, se necessário, da traquéia. Induzir o início da respiração realizando estimulação tátil e ventilação com pressão positiva utilizando balão e máscara ou balão e cânula traqueal, manter a circulação realizando massagem cardíaca, administrar medicações ou fluidos ${ }^{(12)}$.

Mediante as considerações expostas, acreditamos ser oportuno propor este estudo, que teve como objetivo: descrever as práticas assistenciais no atendimento em reanimação neonatal no contexto de um Centro de Parto Normal.

\section{CASUÍSTICA E MÉTODO}

Estudo observacional, transversal, com coleta de dados prospectiva. Foi realizado em um hospital público estadual, de nível secundário, localizado na zona leste da cidade de São Paulo, cuja maternidade foi inaugurada em março de 2002, com estrutura e modelo assistencial de um CPN, segundo recomendação do MS. A proposta assistencial adotada por
Práticas assistenciais em reanimação do recém-nascido no contexto de um centro de parto normal 
Karina Fernandes Amélia Fumiko Kimura

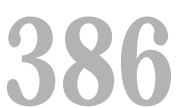

Rev Esc Enferm USP 2005; 39(4):383-90. esta Instituição segue o Programa de Humanização do Parto do Ministério da Saúde ${ }^{(13)}$. A instituição tem como meta implantar o programa Iniciativa Hospital Amigo da Criança (IHAC), proposto pelo Fundo das Nações Unidas para a Infância (UNICEF) e OMS. Para isso, os profissionais estão sendo mobilizados para aderir ao programa incentivando a amamentação precoce e a manutenção do contato mãe-filho, evitando-se, ao máximo, introduzir o aleitamento artificial no período de internação na maternidade. Outra característica do serviço é evitar a medicalização desnecessária bem como fundamentar as tomadas de decisão e implementação de ações de intervenção com base em evidências científicas, em concordância com o Programa de Humanização do Parto e Nascimento.

$\mathrm{O}$ atendimento imediato ao RN é realizado na própria sala em que ocorreu o parto por uma equipe constituída de médicos neonatologistas, enfermeiras obstetras, técnicas e auxiliares de enfermagem.

Constituíram-se nos dados deste estudo os procedimentos de reanimação do RN implementado pelos profissionais no atendimento imediato ao RN de parto normal, cujas mães não apresentaram história de intercorrências na gestação e no trabalho de parto atual.

Para a obtenção do tamanho da amostra $(n)$ foi usada a fórmula proposta por Lemeshow, para a estimação de uma proporção populacional com precisão especificada, considerando-se o intervalo de confiança de 95,0\%, obteve-se uma amostra de 96 atendimentos. A fim de facilitar a análise descritiva, foram obtidos dados de cem atendimentos de nascimentos ocorridos no período de fevereiro a julho de 2003. Foram realizadas as observações dos atendimentos em todos os turnos dos profissionais, que compõem a equipe de atendimento. Os dados foram coletados pela pesquisadora e registrados em um instrumento do tipo check-list, que foi elaborado com base nas práticas descritas pelo MS para atendimento imediato do $\mathrm{RN}^{(11)}$ e nas práticas recomendadas pela OMS em $1996^{(14)}$, adotadas pela Sociedade de Pediatria de São Paulo(15) conforme estabelecidas no guia internacional de reanimação neonatal ${ }^{(16)}$.

Os dados foram armazenados no aplicativo Excel, e para processamento dos mesmos foi utilizado o programa SPSS, versão 10.0. Realizou-se a análise estatística descritiva e, para as variáveis de interesse, a análise inferencial a fim de verificar a dependência entre os dados. As variáveis estudadas e abordadas neste artigo foram: o profissional que assistiu o RN no CPN, o aspecto do líquido amniótico, o procedimento de aspiração das vias aéreas -local aspirado, tipo de ventilação, escore obtido no boletim de Apgar no primeiro e quinto minuto de vida, profissional que atribuiu o índice de Apgar, evolução do RN após receber os cuidados imediatos.

Em observância às determinações da Resolução 196/96(17), antes de iniciar a coleta dos dados, foi solicitada autorização da Instituição para efetuar o estudo. $\mathrm{O}$ projeto de pesquisa foi submetido à apreciação e aprovação do Comitê de Ética em Pesquisa da Instituição onde os dados foram coletados.

\section{RESULTADOS E DISCUSSÃO}

$\mathrm{O}$ atendimento ao RN no CPN deste serviço é realizado por uma equipe multiprofissional, cada qual com suas atribuições determinadas. A Tabela 1 apresenta o profissional que recepcionou o $\mathrm{RN}$ após o nascimento. Neste serviço, o médico neonato-logista coordena o atendimento ao RN. Entretanto, ele não trabalha exclusivamente no CPN, seu serviço é solicitado na iminência da ocorrência de um nascimento. Em decorrência disso, em 36,0\% dos nascimentos, a enfermeira obstetra de plantão recepcionou e realizou os cuidados iniciais do RN até que o neonatologista assumisse a coordenação do atendimento imediato. No CPN algumas práticas são incentivadas, tais como evitar a separação entre mãe e RN, imediatamente após o desprendimento do organismo materno, o RN é mantido em contato pele-a-pele com a mãe para manter a estabilidade térmica, proporcionar a formação da microbiota do $\mathrm{RN}$ e fornecer condições para iniciar o aleitamento materno precoce. Tal prática é estimulada e depende da avaliação das condições de vitalidade do $\mathrm{RN}$ ao nascimento.

Tabela 1 - Profissionais que prestaram assistência imediata ao RN. (São Paulo, 2003)

\begin{tabular}{lcc}
\hline \multicolumn{1}{c}{ Profissional } & N & \% \\
\hline Neonatologista & 64 & 64,0 \\
Enfermeira Obstetra & 36 & 36,0 \\
\hline Total & 100 & 100,0 \\
\hline
\end{tabular}

A Tabela 2 descreve o aspecto do líquido amniótico presente ao nascimento, sendo este um dos sinais que o profissional considera ao avaliar a necessidade de intervir dando início às manobras de reanimação neonatal com o procedimento de aspiração das vias respiratórias. Verifica-se que em $76,0 \%$ dos atendimentos foi encontrado líquido amniótico claro ao nascimento. 
Tabela 2 - Aspecto do líquido amniótico observado nos atendimentos dos RNs. (São Paulo, 2003)

\begin{tabular}{lcc}
\hline Aspecto do líquido amniótico & N & $\%$ \\
\hline Claro com grumos & 76 & 76,0 \\
Mecônio fluido & 14 & 14,0 \\
Mecônio espesso & 10 & 10,0 \\
\hline Total & 100 & 100,0 \\
\hline
\end{tabular}

A aspiração das vias respiratórias foi realizada em $47,0 \%$ dos atendimentos, conforme mostra a Tabela 3, desse total, em 95,7\%, o procedimento foi efetuado pelo neonatologista e em $4,3 \%$ pela enfermeira obstetra.
Tabela 3 - Local aspirado pelos profissionais nos RNs submetidos ao procedimento. (São Paulo, 2003)

\begin{tabular}{lrr}
\hline \multicolumn{1}{c}{ Local aspirado } & N & \multicolumn{1}{c}{$\%$} \\
\hline Cavidade oral & 47 & 100,0 \\
Vias aéreas superiores (VAS) & 47 & 100,0 \\
Estômago & 30 & 63,8 \\
Traquéia & 3 & 6,4 \\
\hline
\end{tabular}

Todos os 47 RNs submetidos ao procedimento de aspiração tiveram a cavidade oral e as vias aéreas superiores aspiradas, $3(6,4 \%)$ RNs submetidos à aspiração traqueal.

A análise inferencial mostrou haver associação estatística significante entre a realização do procedimento de aspiração e a característica do líquido meconial, apresentado pelo RN no nascimento (Tabela 4).

Tabela 4 - Associação entre aspecto do líquido amniótico e realização do procedimento de aspiração por local aspirado. (São Paulo, 2003)

\begin{tabular}{|c|c|c|c|c|c|}
\hline \multirow{2}{*}{$\begin{array}{l}\text { LOCAL } \\
\text { ASPIRADO }\end{array}$} & \multirow{2}{*}{$\begin{array}{l}\text { Procedimento } \\
\text { realizado }\end{array}$} & \multicolumn{3}{|c|}{ Aspecto do líquido amniótico ${ }_{R}$} & \multirow{2}{*}{$\mathrm{p}$ - valor } \\
\hline & & $\begin{array}{c}\text { Claro com } \\
\text { grumos }(\mathrm{N}=76)\end{array}$ & $\begin{array}{l}\text { Meconial fluído } \\
(\mathrm{N}=14)\end{array}$ & $\begin{array}{c}\text { Meconial } \\
\text { espesso }(N=10)\end{array}$ & \\
\hline Cavidade oral & $\begin{array}{l}\text { Não } \\
\text { Sim }\end{array}$ & $\begin{array}{l}49(64,5 \%)_{1,4} \\
27(35,5 \%)_{-1,5}\end{array}$ & $\begin{array}{c}3(21,4 \%)_{1,6} \\
11(78,6 \%)_{1,7}\end{array}$ & $\begin{array}{l}1(10,0 \%)_{-1,9} \\
9(90,0 \%)_{2,1}\end{array}$ & $<0,001^{*}$ \\
\hline VAS & $\begin{array}{l}\text { Não } \\
\text { Sim }\end{array}$ & $\begin{array}{l}49(64,5 \%)_{1,4} \\
27(35,5 \%)_{-1,5}\end{array}$ & $\begin{array}{c}3(21,4 \%)_{-1,6} \\
11(78,6 \%)_{1,7}\end{array}$ & $\begin{array}{l}1(10,0 \%)_{-1,9} \\
9(90,0 \%)_{2,1}\end{array}$ & $<0,001_{\mathrm{F}}$ \\
\hline Estômago & $\begin{array}{l}\text { Não } \\
\text { Sim }\end{array}$ & $\begin{array}{l}58(76,3 \%)_{0,7} \\
18(23,7 \%)_{-1,0}\end{array}$ & $\begin{array}{r}11(21,4 \%)_{1,6} \\
3(21,4 \%)_{-0,6}\end{array}$ & $\begin{array}{l}1(10,0 \%)_{2,3} \\
9(90,0 \%)_{3,5}\end{array}$ & $<0,001 *_{\mathrm{F}}$ \\
\hline Traquéia & $\begin{array}{l}\text { Não } \\
\text { Sim }\end{array}$ & $\begin{array}{r}75(98,7 \%)_{0,1} \\
1(1,3 \%)_{-0,8}\end{array}$ & $\begin{array}{c}14(100,0 \%)^{0,1} \\
0(21,4 \%)_{-0,6}\end{array}$ & $\begin{array}{l}8(80,0 \%)_{-0,5} \\
2(20,0 \%)_{3,1}\end{array}$ & $<0,034^{*}$ \\
\hline
\end{tabular}

* Estatisticamente significante $\mathrm{F}=$ teste exato de Fisher $\mathrm{R}=$ resíduo

A presença de mecônio na traquéia do RN é indicação de que a aspiração ocorreu intra-útero, segundo a Resolução n 196/96 do Conselho Nacional de Saúde ${ }^{(17)}$. Neste estudo, a aspiração traqueal foi realizada em três RNs, sendo que em um deles a técnica empregada foi por introdução da sonda de aspiração de calibre 8, através do laringoscópio e, os outros dois RNs foram submetidos à intubação traqueal para serem aspirados. A Tabela 5 mostra que existe associação estatística significante entre o procedimento de intubação e a presença de líquido meconial espesso.

Tabela 5 - Associação entre aspecto do líquido amniótico e realização do procedimento de intubação. (São Paulo, 2003)

\begin{tabular}{|c|c|c|c|c|}
\hline \multirow{2}{*}{ I ntubação } & \multicolumn{3}{|c|}{ Aspecto do líquido amniótico ${ }_{R}$} & \multirow{2}{*}{$\mathrm{p}$ - valor } \\
\hline & $\begin{array}{c}\text { Claro com } \\
\text { grumos }(\mathrm{N}=76)\end{array}$ & $\begin{array}{l}\text { Meconial fluído } \\
(\mathrm{N}=14)\end{array}$ & $\begin{array}{c}\text { Meconial } \\
\text { espesso }(\mathrm{N}=10)\end{array}$ & \\
\hline Não & $76(100,0 \%)_{0,2}$ & $14(100,0 \%)_{0,1}$ & $8(80,0 \%)_{-0,6}$ & \multirow{2}{*}{$<0,003_{\mathrm{F}}$} \\
\hline Sim & $0 \quad(0 \%)_{-1,5}$ & $0 \quad(0 \%)_{-0,5}^{-, 4}$ & $2(20,0 \%)_{4,0}$ & \\
\hline
\end{tabular}

\footnotetext{
* Estatisticamente significante $\mathrm{F}=$ teste exato de Fisher $\mathrm{R}=$ resíduo
}

Práticas assistenciais em reanimação do recém-nascido no contexto de um centro de parto normal 
Karina Fernandes Amélia Fumiko Kimura
A oxigenação é um procedimento de reanimação neonatal. Neste estudo $26(26,0 \%) \mathrm{RNs}$ foram oxigenados, sendo que $21(80,8 \%)$ foram ventilados apenas com máscara aberta e $5(19,2 \%)$ deles ventilados com máscara aberta e pressão positiva. Dos 26 RNs, que foram oxigenados, $10(38,4 \%)$ permaneceram em observação sob o berço aquecido. Destes 10 RNs, 4(40,0\%) evoluíram bem, porém, 6(60,0\%) desenvolveram desconforto respiratório, sendo encaminhados à UTI neonatal, pois necessitaram de cuidados especiais.

Tabela 6 - Distribuição da freqüência da realização do procedimento de oxigenação. (São Paulo, 2003)

\begin{tabular}{ccc}
\hline Oxigenação & N & $\%$ \\
\hline Não & 74 & 74,0 \\
Sim & 26 & 26,0 \\
\hline Total & 100 & 100,0
\end{tabular}

A associação entre procedimento de oxigenação e aspecto do líquido amniótico mostrou-se estatisticamente significante $(\mathrm{p}<0,05)$.
Um RN (10,0\%) necessitou de massagem cardíaca, pois não conseguiu estabelecer freqüência cardíaca maior que 60bpm, de acordo com o Guia de Reanimação Neonatal ${ }^{(15)}$.

O índice de APGAR é um instrumento que serve para avaliar as condições de nascimento do RN. Avalia a irritabilidade reflexa, a respiração, a coloração da pele, o tônus muscular e a frequiência cardíaca (FC) no primeiro e quinto minuto de vida $^{(18-19)}$. É uma avaliação criticada por muitos autores, porém, continua sendo utilizado na maioria das instituições. Neste estudo, observou-se que a mediana do APGAR de primeiro minuto foi 9 com valor mínimo de 3 e máximo de 10 e o do quinto minuto também 9 , entretanto o menor valor foi 6. No entanto, chamou a atenção que, embora em $36,0 \%$ dos atendimentos o neonatologista estivesse ausente no primeiro minuto de vida do RN, sendo a enfermeira obstetra a assumir os cuidados imediatos com o RN, a atribuição de estabelecer o Apgar do primeiro minuto foi assumida, em 99,0\% dos atendimentos, pelo médico neonatologista.

Tabela 7 - Valores da média, mediana, erro padrão, desvio-padrão, mínimo e máximo. (São Paulo, 2003)

\begin{tabular}{lcccccc}
\hline \multirow{2}{*}{$\begin{array}{l}\text { Tempo de } \\
\text { avaliação do } \\
\text { APGAR }\end{array}$} & $\mathrm{N}$ & Mediana & Erro padrão & $\begin{array}{l}\text { Desvio } \\
\text { padrão }\end{array}$ & Mínimo & Máximo \\
\cline { 2 - 6 } & 100 & 9 & 0,13 & 1,31 & 3 & 10 \\
\hline 1o min. & 100 & 9 & 0,07 & 0,7 & 6 & 10 \\
5o min & & & & & \\
\hline
\end{tabular}

Tabela 8 - Profissionais que atribuíram 0 APGAR do RN. (São Paulo, 2003)

\begin{tabular}{lrc}
\hline \multicolumn{1}{c}{ Profissional } & N & \% \\
\hline Neonatologista & 99 & 99,0 \\
EnfermeiraObstetra & 1 & 1,0 \\
\hline \multicolumn{1}{c}{ Total } & 100 & 100,0 \\
\hline
\end{tabular}

A maioria dos RNs foi encaminhada com suas mães ao Alojamento Conjunto; porém, houve um grupo de RNs que apresentou intercorrências, necessitando de suporte ventilatório e de recursos mais complexos para sua sobrevivência, particularmen- te, no grupo de RNs que nasceu com presença de líquido amniótico meconial espesso, houve maior freqüência de RNs encaminhados à UTI neonatal.

Tabela 9 - Freqüência de RNs que evoluíram com quadro de desconforto respiratório após terem sido submetidos ao atendimento imediato inicial. (São Paulo, 2003)

\begin{tabular}{crc}
\hline Desconforto respiratório & N & $\%$ \\
\hline Não & 94 & $\begin{array}{r}94,0 \\
6,0\end{array}$ \\
Sim & 6 & 6,0 \\
\hline Total & 100 & 100,0 \\
\hline
\end{tabular}


A Tabela 10 mostra a associação estatisticamente significante, $(\mathrm{p}<0,05)$, entre o aspecto do líquido amniótico com a unidade em que o RN foi transferido. RNs impregnados com secreção meconial espessa foram os que apresentaram maior dificuldade de adaptação no período transitivo neonatal, requerendo recursos humanos e técnicos de maior complexidade que a unidade de Alojamento Conjunto não dispõe, sendo encaminhados para a UTI neonatal.

Tabela 10 - Associação entre aspecto do líquido amniótico e unidade de transferência do RN. (São Paulo, 2003)

\begin{tabular}{lcccc}
\hline \multirow{2}{*}{$\begin{array}{l}\text { Unidade de } \\
\text { transferência }\end{array}$} & \multicolumn{3}{c}{ Aspecto do líquido amniótico $\mathrm{N}_{\mathrm{R}}$} & p - valor \\
\cline { 2 - 4 } & grumos (N=76) & $\begin{array}{c}\text { Meconial fluído } \\
(\mathrm{N}=14)\end{array}$ & $\begin{array}{c}\text { Meconial } \\
\text { espesso }(\mathrm{N}=10)\end{array}$ & \\
\hline $\begin{array}{l}\text { Aloj. conjunto } \\
\text { UTI neonatal }\end{array}$ & $\begin{array}{c}73(96,1 \%)_{0,2} \\
(3,9 \%)_{-0,7}\end{array}$ & $\begin{array}{c}14(100,0 \%)_{0,1} \\
(0 \%)_{-0,9}\end{array}$ & $\begin{array}{c}7(70,0 \%)_{-0,8} \\
3(30,0 \%)_{3,1}\end{array}$ & $<0,017_{\mathrm{F}}$ \\
\hline
\end{tabular}

* Estatisticamente significante $\quad \mathrm{F}=$ teste exato de Fisher $\mathrm{R}=$ resíduo

\section{CONCLUSÃOE CONSIDERAÇÕES FINAIS}

A freqüência de RNs submetidos à reanimação, considerando o procedimento de aspiração das vias respiratórias como manobra inicial, ocorreu em $47,0 \%$ dos atendimentos. Deste total, todos tiveram a cavidade oral e as vias aéreas superiores aspiradas, $30(63,8 \%)$ foram submetidos à aspiração do estômago e $3(6,4 \%)$ tiveram a traquéia aspirada, sendo 2 $(66,7 \%)$ por intubação. O procedimento de aspiração apresentou associação estatisticamente significante com a presença e a característica do líquido amniótico observada ao nascimento, indicando que a decisão do profissional para realizar o procedimento é determinada pelas condições de avaliação do estado do RN e da característica do líquido amniótico.

A oxigenoterapia foi empregada em $26,0 \%$ dos atendimentos, havendo associação estatisticamente significante entre a execução desse procedimento com o aspecto do líquido amniótico presente ao nascimento.

A mediana do valor do APGAR obtida no primeiro minuto e quinto minuto foi 9 indicando que os RNs apresentavam-se em boas condições de vitalidade. A atribuição do valor do APGAR ocorreu em 99,0\% dos atendimentos pelo neonatologista, mesmo quando este profissional não esteve presente no momento do nascimento na sala de parto, o que se verificou em $64,0 \%$ dos partos normais, assumindo o atendimento minutos após. Apenas um RN necessitou de massagem cardíaca durante a reanimação.

A maioria dos RNs, 94,0\%, apresentou boa resposta e evolução, sendo encaminhados ao Alojamento Conjunto com a mãe. Apenas $6,0 \%$ não con- seguiram estabilizar-se adequadamente, necessitando de suporte oferecido pela Unidade de Terapia Intensiva Neonatal. Embora não tenha sido objetivo deste estudo, vale informar que os RNs transferidos para a UTI neonatal permaneceram em observação ou tratamento por um período breve, nesta unidade, sendo transferidos para o Alojamento Conjunto, assim que estabilizaram os parâmetros vitais.

Por ser o CPN um modelo assistencial inovador no atendimento à parturiente, concepto e RN, implantado nos serviços de maternidade do Brasil a partir de 1999, mais estudos devem ser realizados nesse contexto para se obter subsídios que possam contribuir para a avaliação do impacto desta proposta nos indicadores de qualidade de serviço, no que diz respeito à redução dos índices de morbidade e mortalidade perinatal e nos custos em recursos materiais e humanos investidos nos serviços de saúde.

Neste estudo, verificou-se que $6,0 \%$ dos RNs após serem submetidos a procedimentos iniciais de reanimação, evoluíram com intercorrências que demandaram suporte ventilatório e transferência para a UTI neonatal. Conforme já mencionado, a literatura aponta que entre 10 a $15 \%$ dos nascimentos, há necessidade de intervenção com o emprego de manobras de reanimação neonatal. Se considerarmos o procedimento de aspiração de vias aéreas como uma manobra de reanimação, a prevalência de RNs submetidos à reanimação neonatal neste estudo foi bastante superior ao citado pela literatura. Este dado aponta que o procedimento de aspiração das vias aéreas é uma prática comum sendo necessário avaliar a sua real necessidade, já que a variável Apgar de primeiro minuto apresentou mediana 9. Entretanto, é necessário que mais estudos sejam realizados focalizando a associação entre as práticas empregadas em reanimação neonatal, o tipo de parto,
Práticas assistenciais em reanimação do recém-nascido no contexto de um centro de parto norma 
Karina Fernandes Amélia Fumiko Kimura idade gestacional e os resultados perinatais obtidos. Tais estudos poderão contribuir para avaliar o impacto dos modelos e práticas adotadas nas taxas de morbimortalidade neonatal, fornecendo sub- sídios para estabelecer evidências científicas que apontem para as práticas mais seguras e efetivas que contribuam para a redução dessa taxas.

\section{REFERÊNCIAS}

(1) Kenner C, editor. Enfermagem neonatal. $2^{\mathrm{a}}$ ed. Rio de Janeiro: Reichmann \& Affonso; 2001. Adaptação do neonato; p. 1-29.

(2) Askin DF. Complications in the transition from fetal to neonatal life. J Obstect Gynecol Neonatal Nurs 2002; 31(3):318-27.

(3) Janicas RCSV. Identificação dos fatores intervenientes na implementação da prática do contato corporal precoce entre mãe e recém-nascido em uma maternidade pública. [dissertação] São Paulo (SP): Escola de Enfermagem da USP; 2001

(4) Maranhão G, Joaquim ACK, Siu C. A mortalidade perinatal e neonatal no Brasil. [monografia online] Brasília: Ministério da Saúde; 1998. Disponível em: <http://www. saude.gov.br/programa/scriança/publica. htm>(30 ago. 2000)

(5) Victora CG, Barros FC, Vaughan JP. Epidemiologia da desigualdade. São Paulo: Hucitec; 1988.

(6) Gray RH, Ferraz EM, Amorim MS. Levels and determinant of early neonatal mortality in Natal, Northeastern Brazil: results of a surveillance and casecontrol study. Int J Epidemiol 1991; 76(3):200-6.

(7) Aerts DRGCA. Investigação dos óbitos perinatais e infantis: seu uso no planejamento de políticas públicas de saúde. J Pediatr 1997; 73(6):364-6.

(8) Ribeiro VS, Silva AAM. Tendências da mortalidade neonatal em São Luís, Maranhão, Brasil, de 1979 a 1996. Cad Saúde Pública 2000; 16(2):429-38.

(9) Leite AJM, Marcopito LF, Diniz RLP, Silva AVS, Souza LCB, Borges JC et al. Mortes perinatais no município de Fortaleza, Ceará: o quanto é possível evitar? J Pediatr 1997; 73(6):388-94.

(10) Ministério da Saúde. Portaria n. 985/GM, de 5 de agosto de 1999. Dispõe sobre criação, normas, critérios, atribuições, definições das características físicas e recursos humanos para o funcionamento do Centro de Parto Normal - CPN no âmbito do SUS. Diário Oficial da República Federativa do Brasil, Brasília, 6 ago. 1999. Seção 1, p. 51.

(11) Ministério da Saúde. Secretaria de Políticas de Saúde. Área Técnica de Saúde da Mulher. Parto, aborto e puerpério: assistência humanizada à mulher. Brasília; 2001.

(12) Almeida MFB, Guinsburg R. Controvérsias em reanimação do recém-nascido. J Pediatr 2001; 77(Supl.1):S41-S52.
(13) Ministério da Saúde. Secretaria Executiva. Programa humanização do parto: humanização do pré-natal e nascimento. Brasília; 2000.

(14) Organização Mundial da Saúde (OMS). Maternidade segura: assistência ao parto normal: um guia prático. Genebra; 1996.

(15) Guinsburg R, Almeida MFB. Revisão: reanimação neonatal: atualização 2001. São Paulo; 2001. [Apostila do Curso de Reanimação Neonatal - Sociedade de Pediatria de São Paulo].

(16) Niermeyer S, Kattwinkel J, Van Reempts P, Nadkarni $\mathrm{V}$, Phillips B, Ziderman D et al. International guidelines for neonatal resuscitation: an excerpt from the guidelines 2000 for cardiopulmonary resuscitation and emergency cardiovascular care: international consensus on science. Pediatrics [serial online] 2000; 106(03). Available from: <http://www. pediatrics.org/cgi/content/full/106/3/e29> (18 Aug. 2002)

(17) Conselho Nacional de Saúde. Resolução n. 196 de 10 de outubro de 1996. Dispõe sobre as diretrizes e normas regulamentadoras de pesquisas envolvendo seres humanos. Bioética 1996; 4(2 Supl):15-25.

(18) Cunningham FG, MacDonald PC, Gant NF, Leveno KJ, Gilstrap LC, Hankins GDV et al. Williams Obstetrícia. 20a ed. Rio de Janeiro: Guanabara Koogan; 2000. O recém-nascido; p. 346-59.

(19) World Health Organization (WHO). Basic newborn resuscitation: a practical guide. [online] 1998. Available from: <http://:www.who.int/reproductivehealth/publications/msm 981 table of contentes. em.html> (14 Oct. 2003) 